SU-4252-886

DIAS-STP-09-12

PI-other-148

\title{
Poincaré Quasi-Hopf Symmetry and Non-Associative Spacetime Algebra from Twisted Gauge Theories
}

\author{
A. P. Balachandran ${ }^{a, b * \dagger}$ and B. A. Qureshi ${ }^{c, d \ddagger}$ \\ ${ }^{a}$ Department of Physics, Syracuse University, Syracuse NY, 13244-1130, USA. \\ ${ }^{b}$ Departamento de Matemáticas, Universidad Carlos III de Madrid, 28911 Leganés, Madrid, Spain. \\ ${ }^{c}$ School of Theoretical Physics, Dublin Institute for Advanced Studies, Dublin 4, Ireland. \\ $d$ Perimeter Institute for Theoretical Physics, Waterloo, Ontario, N2L2Y5, Canada
}

\begin{abstract}
In previous work, starting from the Moyal plane, we formulated interacting theories of matter and gauge fields with only the former fields twisted. In this approach, gauge theories, including the standard model, can be formulated without new gauge degrees of freedom. We show their underlying symmetry algebra to be Poincaré quasi-Hopf . The associated spacetime algebra is hence non-associative.
\end{abstract}

\section{Introduction}

When spacetime is noncommutative, it is often the case that diffeomorphisms do not act as a group of automorphisms of this algebra. Instead it can be the case that symmetries act on the spacetime algebra as a Hopf or a quasi-Hopf algebra [1-3]. A prominent example is provided by the Groenewald-Moyal (GM) plane $\mathcal{A}_{\theta}\left(\mathbb{R}^{d}\right)$ and the Poincaré symmetry. The algebra $\mathcal{A}_{\theta}\left(\mathbb{R}^{d}\right)$ is the algebra of functions on $\mathbb{R}^{d}$ with the "*" product

$$
\begin{gathered}
f_{1} * f_{2}=f_{1} e^{\frac{i}{2} \overleftarrow{\partial_{\mu}} \theta^{\mu \nu} \vec{\partial}_{\nu}} f_{2}, \\
f_{i} \in \mathcal{A}_{\theta}\left(\mathbb{R}^{d}\right), \theta^{\mu \nu}=-\theta^{\nu \mu}=\text { constant. }
\end{gathered}
$$

Let $\hat{\mathcal{P}}_{+}^{\uparrow}$ be the standard universal cover of the (let us say) the connected Poincaré group. Then $\hat{\mathcal{P}}_{+}^{\uparrow}$ does not act as a standard group of automorphisms on $\mathcal{A}_{\theta}\left(\mathbb{R}^{d}\right)$ since $\theta^{\mu \nu}$ are constants. There is however a Hopf algebra $\left(\mathbb{C} \hat{\mathcal{P}}_{+}^{\uparrow}, \Delta_{\theta}\right)$ where $\mathbb{C} \hat{\mathcal{P}}_{+}^{\uparrow}$ is the group algebra of $\hat{\mathcal{P}}_{+}^{\uparrow}$ and $\Delta_{\theta}$ is a deformed coproduct:

$$
\begin{aligned}
\Delta_{\theta}(g) & =F_{\theta}^{-1} g \otimes g F_{\theta}, g \in \hat{\mathcal{P}}_{+}^{\uparrow}, \\
F_{\theta} & =e^{\frac{i}{2} \partial_{\mu} \theta^{\mu \nu} \otimes \partial_{\nu}}=\text { Drinfel'd's twist factor } .
\end{aligned}
$$

\footnotetext{
*Cátedra de Excelencia

†bal@phy.syr.edu

${ }^{\ddagger}$ bqureshi@stp.dias.ie
} 
(We do not include the counit $\epsilon$ and the antipode $S$ in the notation for simplicity).

The twist of coproduct implies the twist of statistics as well. Its effects can be accounted for by "dressing" [4-6] the quantum field $\phi_{0}$ of matter for $\theta^{\mu \nu}=0$ :

$$
\begin{aligned}
\phi_{\theta} & =\text { quantum field of matter for noncommutativity parameter } \theta^{\mu \nu} \\
& =\phi_{0} e^{\frac{1}{2} \overleftarrow{\partial_{\mu}} \theta^{\mu \nu} P_{\nu}}, P_{\nu}=\text { Total momentum of all fields. }
\end{aligned}
$$

(Although here we focus on just one field, this formula is valid in an interacting field theory with many fields. Then $P_{\nu}$ refers to the full four-momentum of the interacting field theory.)

Hereafter $A \wedge B$ will denote $A_{\mu} \theta^{\mu \nu} \otimes B_{\nu}$.

A remarkable feature of the dressing transformation is its self-reproducing property:

$$
\phi_{\theta} * \chi_{\theta}=\left(\phi_{0} \chi_{0}\right) e^{\frac{1}{2} \overleftarrow{\partial} \wedge P}
$$

In particular, for the interaction Hamiltonian density, it implies [7,8] that,

$$
\mathcal{H}_{I}^{\theta}=\mathcal{H}_{I}^{0} e^{\frac{1}{2} \overleftarrow{\partial} \wedge P}
$$

and that the interaction representation $S$-operator is independent of $\theta^{\mu \nu}, S_{\theta}=S_{0}$. But scattering amplitudes show time delays which depend on $\theta^{\mu \nu}$ [7, 9 .

The above approach has no major physical problems in the absence of gauge fields. When gauge fields are introduced, new issues arise. In the covariant derivative, $D_{\mu}=\partial_{\mu}+A_{\mu}$, at first sight, it seems natural to regard $A_{\mu}$ as $\underline{G}$-valued functions on $\mathcal{A}_{\theta}\left(\mathbb{R}^{d}\right)$ where $\underline{G}$ is the Lie algebra of the compact simple group $G$ underlying the gauge theory. Unfortunately, as is well-known, this point of view cannot be sustained, since $\left[D_{\mu}, D_{\nu}\right]$ is valued in the enveloping algebra $\mathcal{U}(\underline{G})$ of $\underline{G}$. If we work in an $N$-dimensional irreducible representation of $\underline{G},\left[D_{\mu}, D_{\nu}\right]$ is generally valued in $U(N)$. One may thus be obliged to introduce new gauge fields [10] causing problems in formulating for example the standard model on $\mathcal{A}_{\theta}\left(\mathbb{R}^{d}\right)$.

We remark however that new gauge degrees of freedom may not be necessary. Vassilevich 11 has found new gauge invariant expressions which vanish as $\theta^{\mu \nu} \rightarrow 0$ and which can be added to the action. With their inclusion, it may be possible to avoid new gauge degrees of freedom.

In past work [12, 13], we developed an alternative formulation. There the gauge fields $A_{\mu}$ are $\underline{G}$-valued functions on the commutative algebra $\mathcal{A}_{0}\left(\mathbb{R}^{d}\right)$. The fields $A_{\mu}$ are thus not twisted: $A_{\mu}^{\theta}=A_{\mu}^{0}$. Matter fields are still based on $\mathcal{A}_{\theta}\left(\mathbb{R}^{d}\right)$ and are given by $\phi_{\theta}$ where $P_{\nu}$ is now the total momentum including that of gauge fields.

Such a formulation is possible since $\mathcal{A}_{\theta}\left(\mathbb{R}^{d}\right)$ is an $\mathcal{A}_{0}\left(\mathbb{R}^{d}\right)$-module. It has specific consequences such as the appearance of new types of diagrams, UV-IR mixing of a new sort and CPT violation [13-16].

Thus gauge fields are based on the commutative algebra of functions $\mathcal{A}_{0}\left(\mathbb{R}^{d}\right)$. Hence Poincaré transformations act on gauge fields with the untwisted coproduct $\Delta_{0}$. The corresponding Poincaré Hopf algebra is $\left(\mathbb{C} \hat{\mathcal{P}}_{+}^{\uparrow}, \Delta_{0}\right)$ whereas it is $\left(\mathbb{C} \hat{\mathcal{P}}_{+}^{\uparrow}, \Delta_{\theta}\right)$ for matter fields. (The hat on Poincaré is to show that we deal with its covering group.)

As gauge and matter fields interact, the existence of two different Poincaré Hopf algebras raises consistency questions regarding our treatment of Poincaré symmetry. In this paper, 
we formulate a single Poincaré quasi-Hopf symmetry acting on both matter and gauge fields [17-20]. The coproduct on this symmetry algebra is not coassociative. As a result, the product on the spacetime algebra is not associative. The statistics group too is changed: it is neither the permutation nor the braid group.

Quasi-Hopf algebras were formulated by Drinfel'd. They were later studied by Mack, Schomerus [17-19], Majid [20] and others. But perhaps it is here that they appear for the first time in the context of relativistic quantum field theories.

In this note, we describe the preceding new results indicating all the necessary steps. But there are several aspects not elaborated here such as the properties of the $\mathcal{R}$-matrix and the construction of "covariant products of quantum fields" [17-19]. Elsewhere we will give a full treatment basing our considerations on the work of Mack and Schomerus [17-19]. But, for now, in the interests of simplicity, we highlight just the main points.

This paper has been written with the Lehmann-Symanzik-Zimmermann (LSZ) formalism of quantum field theories (qft's) on $\mathcal{A}_{\theta}\left(\mathbb{R}^{d}\right)$ in [21] in mind. It works with interacting fields and total energy-momentum operators $P_{\mu}$ which include interactions. But it is easily adapted to the perturbative approach of [12] by replacing $P_{\mu}$ by their free-field counterparts.

\section{The Drinfel'd Twists and Quasi-Hopf Algebras}

Drinfel'd gives a general procedure to obtain new Hopf algebras starting from a given Hopf algebra using twists. The construction of the coproduct $\Delta_{\theta}$ is an example of this general theory of twisting.

This section follows the treatment of Drinfel'd's work as given in [20]. We always assume that a quasitriangular structure (the $\mathcal{R}$-matrix) exists. Here we only give the definitions and properties which are essential to follow the later sections for completeness. For details, see $[20]$.

Consider a Hopf algebra $H$ with a coproduct $\Delta$, which acts in another algebra $\mathcal{A}$ with multiplication map $m_{0}$. Now consider an invertible element $F \in H \otimes H$ ( the twist element) which is a counital 2-cocycle ( a condition which we will describe shortly). Then one can define a new Hopf algebra with the same algebra structure as $H$, but with the new coproduct

$$
\Delta_{F}=F^{-1} \Delta F
$$

and this algebra acts in a new carrier algebra $\mathcal{A}_{F}$ where the multiplication rule is now given by

$$
m_{F}=m_{0} F .
$$

The new coproduct is generally not cocommutative (even if the original untwisted coproduct is) i.e. if we flip the entries in the tensor product which appears in $\Delta_{F}(\cdot)$, we do not get back the original coproduct:

$$
\Delta_{F}^{\prime} \equiv s \Delta_{F} \neq \Delta_{F}
$$

where $s$ is the transposition map which flips the entries in the tensor product in the coproduct. Hence the usual symmetrization/antisymmetrization in the tensor products of the carrier algebra ( that is, the statistics) is not compatible with the coproduct. Rather, in 
any theory with multiparticle states, the statistics is governed by the $\mathcal{R}$-matrix associated with the coproduct.

The $\mathcal{R}$-matrix has the property

$$
\mathcal{R} \Delta=\Delta^{\prime} \mathcal{R}
$$

Therefore the correct statistics operator $\tau$ on $\mathcal{A} \otimes \mathcal{A}$ which is compatible with a general coproduct is given by

$$
\tau=\sigma \circ(\rho \otimes \rho)(\mathcal{R}) .
$$

Here $\sigma$ is the flip operator on the tensor product $V \otimes V$ of representation carrier space $V$ and $\rho$ is a representation by which $H$ acts in $V$. The diagonalization with respect to $\tau$ gives states which are superselected.

It is easy to see that the $\mathcal{R}$-matrix for the coproduct obtained by twisting procedure from a trivial coproduct is given by

$$
\mathcal{R}=F_{21}^{-1} F,
$$

where

$$
F_{21}^{-1}=s F^{-1} .
$$

where $s$ again flips the entries in the tensor product of $F^{-1}$. So $\tau$ can be written as

$$
\tau=\sigma \circ(\rho \otimes \rho)\left(F_{21}^{-1} F\right) .
$$

We will often omit the representation symbol $\rho$ when it is clear from the context.

Thus we see that the twisting procedure works at three levels. It not only twists the coproduct of the symmetry group and the product in the spacetime algebra, but it also changes the usual bosonic/fermionic statistics to twisted bosonic/fermionic statistics.

\subsection{Coassociativity and Quasi-Hopf Agebras}

The coassociativity of coproduct is defined by

$$
(i d \otimes \Delta) \Delta=(\Delta \otimes i d) \Delta .
$$

By duality, this represents the associativity of the carrier algebra 22$]$.

Drinfel'd has defined more general algebraic structures where the above condition fails to hold, called quasi-Hopf algebras. However, this failure is controlled by an intertwiner $\phi \in H \otimes H \otimes H$ ( fulfilling certain properties which we will not discuss) such that

$$
(i d \otimes \Delta) \Delta(h)=\phi((\Delta \otimes i d) \Delta(h)) \phi^{-1}
$$

for all $h \in H$. The definitions for antipode. counit and quasi-triangular structure are also appropriately modified. But we will not discuss those here as well. It is (2.10), that is the central element leading to the definition of quasi-Hopf algebras.

These quasi-Hopf algebras can actually be obtained by twisting with a twist element $F$ which is required to be counital i.e.,

$$
(i d \otimes \epsilon) F=(\epsilon \otimes i d) F=\mathbb{1}
$$


where $\epsilon$ is the counit. It is the 2-cocycle condition on the twist element $F$,

$$
(F \otimes \mathbb{1}) \cdot(\Delta \otimes i d)(F)=(\mathbb{1} \otimes F) \cdot(i d \otimes \Delta)(F),
$$

which ensures the coassociativity of the twisted coproduct. If the twist element $F$ does not fulfill this condition, the resulting Hopf algebra is only a quasi-Hopf algebra. Notice that $F$ only needs to obey (2.11) to qualify as a twist for a resulting quasi-Hopf algebra.

It is important to note that even in a quasi-Hopf algebra, the $\mathcal{R}$ - matrix still obeys (2.4) and is still obtained via (2.6) from the twist operator $F$. Hence the twisted statistics for a twisted quasi-Hopf algebra is again given by

$$
\tau=\sigma F_{21}^{-1} F .
$$

Where we omitted the symbol $\circ$ after $\sigma$.

In general, a quasi-Hopf algebra is a complicated object. However, if it is obtained from a twist $F$, it is easy to use it as all the structures of quasi-Hopf algebras follow from this twist.

\section{The Twisted Fields}

Twisted fields such as $\phi_{\theta}$ contain all the information on statistics, and hence the coproduct on the symmetry algebra $\mathbb{C} \hat{\mathcal{P}}_{+}^{\uparrow}$ and the product on spacetime algebra. This is fully explained in [13, 23]. Therefore we first focus on a uniform construction of the twisted fields. For this purpose, we have to enlarge $\mathbb{C} \hat{\mathcal{P}}_{+}^{\uparrow}$ by introducing a central element $u$. We call the extended algebra as $\overline{\mathbb{C} \hat{\mathcal{P}}_{+}^{\uparrow}}$.

The central element $u$ is effectively a grading operator for the quantum fields. It behaves

like a pure group element under the coproduct $\overline{\Delta_{\theta}}$, counit $\bar{\epsilon}$ and antipode $\bar{S}$ of the extended algebra. Thus

$$
\begin{aligned}
\overline{\Delta_{\theta}}(u) & =u \otimes u, \\
\bar{\epsilon}(c) & =\mathbb{1} \\
\bar{S}(u) & =u^{-1} .
\end{aligned}
$$

The *-operator on $\mathbb{C} \hat{\mathcal{P}}_{+}^{\uparrow}$ is extended to $\overline{\mathbb{C} \hat{\mathcal{P}}_{+}^{\uparrow}}$ by setting

$$
u^{*} u=u u^{*}=\mathbb{1} .
$$

It is thus a unitary element.

Let $\chi_{0}^{g}$ and $\chi_{0}^{m}$ generically denote a basic untwisted gauge and matter field. The element $u$ acts on the fields by conjugation as usual. This representation of $u$ on fields is denoted by $A d$. Thus

$$
A d u \chi_{0}^{g, m}:=u \chi_{0}^{g, m} u^{-1}
$$

We set

$$
A d u \chi_{0}^{g}=+\chi_{0}^{g}, \quad A d u \chi_{0}^{m}=-\chi_{0}^{m} .
$$

Thus $u$ is a grading operator with $\chi_{0}^{g}$ being even and $\chi_{0}^{m}$ being odd. 
We complete the definition of $u$ in quantum field theory by setting $u=\mathbb{1}$ on vacuum:

$$
u|0\rangle=|0\rangle .
$$

It follows from (3.6) that

$$
\delta_{A d u,-1} \equiv \frac{1}{2}[\mathbb{1}-A d u]
$$

acts as 0 on $\chi_{0}^{g}$ and identity on $\chi_{0}^{m}$ :

$$
\delta_{A d u,-1} \chi_{0}^{g}=0, \delta_{A d u,-1} \chi_{0}^{m}=\chi_{0}^{m} .
$$

It is thus a projector. We avoid the use of $P$ in denoting it as $P$ stands for the momentum operator elsewhere.

We set as usual

$$
\begin{aligned}
A d \Delta_{0}(u) & \equiv(A d \otimes A d)(u \otimes u) \\
& =A d u \otimes A d u .
\end{aligned}
$$

We now write the twisted field $\chi_{\theta}^{g, m}$, which can be matter or gauge, as

$$
\chi_{\theta}^{g, m}=\chi_{0}^{g, m} e^{\frac{1}{2} \overleftarrow{\delta} \wedge P\left(\overleftarrow{\delta_{A d u,-1}}\right)}
$$

where the left arrow indicates action on $\chi_{0}^{g, m}$.

In view of (3.9),

$$
\chi_{\theta}^{g}=\chi_{0}^{g}, \chi_{\theta}^{m}=\chi_{0}^{m} e^{\frac{1}{2} \overleftarrow{\partial} \wedge P} .
$$

These are exactly what we want.

The representation $A d$ extends to $\chi_{\theta}^{g, m}$ in a natural way:

$$
A d u \chi_{\theta}^{g, m}=u \chi_{\theta}^{g, m} u^{-1} .
$$

Remark:

The introduction of a new element to convert a symmetry algebra into a Hopf algebra has occurred before. Thus the SUSY algebra is not Hopf. Now let $N_{F}$ be the fermion number and consider $(-1)^{N_{F}}$. It is the grading operator, commuting with even and anticommuting with odd SUSY generators. Mack and Schomerus [17] extend SUSY to SUSY by including

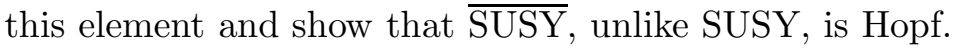

\section{The Coproduct $\bar{\Delta}_{\theta}$ on $\overline{\mathbb{C} \hat{\mathcal{P}}_{+}^{\uparrow}}$}

In the previous section, we did not specify the twisted coproduct $\overline{\Delta_{\theta}}$ on $\mathbb{C} \hat{\mathcal{P}}_{+}^{\uparrow} \subset \overline{\mathbb{C} \hat{\mathcal{P}}_{+}^{\uparrow}}$. We take up that task here.

We know that the coproduct on the gauge sector is just the usual coproduct without any twist,

$$
\left.\overline{\Delta_{\theta}}\right|_{\text {Gauge fields }}=\left.\Delta_{0}\right|_{\text {Gauge fields }},
$$

where

$$
\Delta_{0}(g)=g \otimes g
$$


for a Poincaré group element $g \in \overline{\mathbb{C} \hat{\mathcal{P}}_{+}^{\uparrow}}$. For the matter sector, the coproduct is given by,

$$
\begin{aligned}
\left.\overline{\Delta_{\theta}}\right|_{\text {Matter fields }} & =\Delta_{\theta}=F_{\theta}^{-1} \Delta_{0} F_{\theta}, \\
F_{\theta} & =e^{-\frac{i}{2} P_{\mu} \theta^{\mu \nu} \otimes P_{\nu}} .
\end{aligned}
$$

We want to write a coproduct which reduces to the corresponding coproducts on each sector using a single twist operator:

$$
\overline{\Delta_{\theta}}=\overline{\mathfrak{F}}^{-1} \Delta_{0} \overline{\mathfrak{F}_{\theta}}
$$

In this way we will be defining a new Hopf symmetry structure on the full theory. The twist operator $\overline{\mathfrak{F}_{\theta}}$ which does this job is given by

$$
\overline{\mathfrak{F}_{\theta}}=e^{-\frac{i}{2} P_{\mu} \theta^{\mu \nu} \otimes P_{\nu}\left(\delta_{A d u,-1} \otimes \mathbb{1}\right)} .
$$

It reduces to corresponding twist factors in the respective sectors.

We can do a check that this is indeed the twist factor for our coproduct. We know that for a field $\phi$ to carry a representation of any coproduct $\Delta$, it must fulfill

$$
U(g) \phi=\phi(U \otimes \overleftarrow{\rho})(i d \otimes S) \Delta(g)
$$

where $S$ in the antipode (inverse for pure group elements), $U(g)$ is the operator representative of $g$ on the Hilbert space and $\rho$ is the representation of the group on the field $\phi:$

$$
(\rho(g) \phi)(x)=\phi\left(g^{-1} x\right), \quad g \in \hat{\mathcal{P}}_{+}^{\uparrow} .
$$

The argument for $\rho$ comes from the second factors in $(i d \otimes S) \Delta(g)$. They act as usual from left to right in $\phi$. For the untwisted coproduct $\Delta_{0}=g \otimes g$, (4.7) produces the standard result,

$$
U(g) \phi(x) U(g)^{\dagger}=\phi(g x)
$$

In previous papers [13,23, we have shown that the usual expressions for operators $U(g)$ in terms of untwisted oscillators fulfill equation (4.7) with twisted coproduct when acting on the twisted fields. Thus one knows that the algebraic structure in $\overline{\mathbb{C} \hat{\mathcal{P}}_{+}^{\uparrow}}$ is not changed by changing the coproduct. Also, since the operators act on the same Hilbert space as before, it is expected that the operators $U(g)$ do not change when written in terms of untwisted oscillators because otherwise they will not satisfy the $\overline{\mathbb{C} \hat{\mathcal{P}}_{+}^{\uparrow}}$ algebra. But the remarkable fact is that when they act on twisted fields, they reproduce the twisted coproduct. In other words, the transformations of the twisted fields with correct coproduct can be obtained by simply transforming the untwisted field and $P$ 's in the standard manner.

Let us show this for spinless fields and $g$ a Lorentz transformation. The results for more general fields follow easily. Consider the twisted field $\chi_{\theta}^{g, m}$ given by

$$
\chi_{\theta}^{g, m} \equiv \chi_{0}^{g, m} e^{\frac{1}{2} \overleftarrow{\delta} \wedge P\left(\overleftarrow{\delta}_{A d u,-1}\right)}
$$

We can explicitly write the generators of $\hat{\mathcal{P}}_{+}^{\uparrow}$ in terms of in ( or out) fields. Thus since $P_{\mu}$ is time-independent, at least formally, we have, on letting $x_{0} \longrightarrow-\infty$,

$$
\chi_{\theta}^{g, m, i n}=\chi_{0}^{g, m, i n} e^{\frac{1}{2} \overleftarrow{\partial} \wedge P\left(\overleftarrow{\delta}_{A d u,-1}\right)}
$$


The Poincaré generators ( of the fully interacting theory) have the expansions as in the free field case, but in terms of $\chi_{0}^{i n}$. Therefore we can calculate how $\chi_{\theta}^{g, m, i n}$ transforms and that is enough to find the coproduct on $\mathbb{C} \hat{\mathcal{P}}_{+}^{\uparrow}$.

Now, acting by $U(g)$ on $\chi_{\theta}^{g, m, i n}$ and transforming the untwisted field $\chi_{0}^{g, m, i n}$ and $P_{\mu}$ in the standard way, we have, for $g$ a Lorentz transformation

$$
\begin{aligned}
U(g) \chi_{\theta}^{g, m, i n}(x) & =U(g) \chi_{0}^{g, m, i n}(x) e^{\frac{1}{2} \overleftarrow{\delta} \wedge P\left(\overleftarrow{\delta}_{A d u,-1}\right)} \\
& =\chi_{0}^{g, m, i n}(g x) U(g) e^{\frac{1}{2} \overleftarrow{\partial} \wedge P\left(\overleftarrow{\delta}_{A d u,-1}\right)} \\
& =\chi_{0}^{g, m, i n}(g x) e^{\frac{1}{2}(\overleftarrow{g \partial}) \wedge P\left(\overleftarrow{\delta}_{A d u,-1}\right)} e^{-\frac{1}{2}(\overleftarrow{g \partial}) \wedge P\left(\overleftarrow{\delta}_{A d u,-1}\right)} U(g) e^{\frac{1}{2} \overleftarrow{\partial} \wedge P\left(\overleftarrow{\delta}_{A d u,-1}\right)} \\
& =\chi_{\theta}^{g, m, i n}(g x) e^{-\frac{1}{2}(\overleftarrow{g \partial}) \wedge P\left(\overleftarrow{\delta}_{A d u,-1}\right)} U(g) e^{\frac{1}{2} \overleftarrow{\delta} \overleftarrow{\delta}\left(\overleftarrow{\delta}_{A d u,-1}\right)}
\end{aligned}
$$

Now if we recall that on any field $\phi$, the representation is

$$
\rho\left(g^{-1}\right) \phi(x)=\phi(g x), \quad \rho\left(P_{\mu}\right) \phi(x)=i \partial_{\mu} \phi(x),
$$

then (4.15) is exactly same as (4.7) with $\Delta_{\theta}={\overline{\mathfrak{F}_{\theta}}}^{-1}(g \otimes g) \overline{\mathfrak{F}_{\theta}}$. ( Note that $S$ is an antihomomorphism).

\section{On Lack of Coassociativity of Coproduct $\overline{\Delta_{\theta}}$}

The coproduct $\overline{\Delta_{\theta}}$ is not coassociative. We can see this by evaluating $\left(i d \otimes \overline{\Delta_{\theta}}\right) \overline{\Delta_{\theta}}(g)$ and $\left(\overline{\Delta_{\theta}} \otimes i d\right) \overline{\Delta_{\theta}}(g)$ on vectors $e_{p} \otimes e_{q} \otimes e_{r} \in V_{\text {Gauge }} \otimes V_{\text {Matter }} \otimes V_{\text {Matter }}$ where $V_{\text {Gauge }}$ and $V_{\text {Matter }}$ denote vector spaces with $u=1$ and $u=-1$ and $e_{k} \quad(k=p, q, r)$ denote plane wave vectors $: e_{k}(x)=e^{i k \cdot x}$. Hence $P_{\mu} e_{k}=k_{\mu} e_{k}$.

Consider $\overline{\Delta_{\theta}}(g)$ :

$$
\begin{aligned}
\overline{\Delta_{\theta}(g)}= & e^{\frac{i}{2} P_{\mu} \theta^{\mu \nu} \otimes P_{\nu}\left(\delta_{u,-1} \otimes \mathbb{1}\right)}(g \otimes g) \\
& e^{-\frac{i}{2} P_{\mu} \theta^{\mu \nu} \otimes P_{\nu}\left(\delta_{u,-1} \otimes \mathbb{1}\right)} \\
= & (g \otimes g) e^{\frac{i}{2}(\Lambda(g) P)_{\mu} \theta^{\mu \nu} \otimes(\Lambda(g) P)_{\nu}\left(\delta_{u,-1} \otimes \mathbb{1}\right)} \\
& e^{-\frac{i}{2} P_{\mu} \theta^{\mu \nu} \otimes P_{\nu}\left(\delta_{u,-1} \otimes \mathbb{1}\right)}
\end{aligned}
$$

where $\Lambda: g \longrightarrow \Lambda(g)$ is the homomorphism from $S L(2, C)$ to $\mathcal{L}_{+}^{\uparrow}$.

Now apply id $\otimes \overline{\Delta_{\theta}}$ on the above vectors and collect the exponentials with no $\Lambda(g) P$. They come from the last term :

$$
\begin{aligned}
\left(i d \otimes \overline{\Delta_{\theta}}\right) & e^{-\frac{i}{2} P_{\mu} \theta^{\mu \nu} \otimes P_{\nu}\left(\delta_{u,-1} \otimes \mathbb{1}\right)} \\
= & \exp \left\{-\frac{i}{2} P_{\mu} \otimes\left(\mathbb{1} \otimes \theta^{\mu \nu} P_{\nu}+\theta^{\mu \nu} P_{\nu} \otimes \mathbb{1}\right) \times\right. \\
& \left.\times\left(\delta_{u,-1} \otimes \mathbb{1} \otimes \mathbb{1}\right)\right\} .
\end{aligned}
$$


Applying this to $e_{p} \otimes e_{q} \otimes e_{r} \in V_{\text {Gauge }} \otimes V_{\text {Matter }} \otimes V_{\text {Matter }}$, where the $V$ 's denote the vector spaces for gauge fields or matter as indicated by subscripts,

$$
\text { left-hand side acting on } e_{p} \otimes e_{q} \otimes e_{r}=e_{p} \otimes e_{q} \otimes e_{r}
$$

Also

$$
\begin{aligned}
\left(\overline{\Delta_{\theta}} \otimes i d\right) & e^{-\frac{i}{2} P_{\mu} \theta^{\mu \nu} \otimes P_{\nu}\left(\delta_{u,-1} \otimes \mathbb{1}\right)} e_{p} \otimes e_{q} \otimes e_{r} \\
= & \exp \left\{-\frac{i}{2}\left(P_{\mu} \otimes \mathbb{1}+\mathbb{1} \otimes P_{\mu}\right) \otimes \theta^{\mu \nu} P_{\nu} \times\right. \\
& \left.\times\left(\delta_{(u \otimes u,-1)} \otimes \mathbb{1}\right)\right\} e_{p} \otimes e_{q} \otimes e_{r} \\
= & e^{-\frac{i}{2}(p+q)_{\mu} \theta^{\mu \nu} r_{\nu}} e_{p} \otimes e_{q} \otimes e_{r}
\end{aligned}
$$

so that

$$
\left(i d \otimes \overline{\Delta_{\theta}}\right) \overline{\Delta_{\theta}} \neq\left(\overline{\Delta_{\theta}} \otimes i d\right) \overline{\Delta_{\theta}} .
$$

\section{The Algebra of Functions}

Let us denote it by $\mathcal{B}_{\theta}\left(\mathbb{R}^{N}\right)$. It has two components, with gradings $u=+1$ and -1 :

$$
\mathcal{B}_{\theta}\left(\mathbb{R}^{N}\right)=\mathcal{B}_{\theta}^{+1}\left(\mathbb{R}^{N}\right) \oplus \mathcal{B}_{\theta}^{-1}\left(\mathbb{R}^{N}\right) .
$$

The $*$-product on functions $\alpha, \beta \in \mathcal{B}_{\theta}\left(\mathbb{R}^{N}\right)$ is

$$
\alpha * \beta=m_{0}\left[\overline{F_{\theta}} \alpha \otimes \beta\right]
$$

where $m_{0}$ is the point-wise multiplication map and

$$
\overline{F_{\theta}}=e^{\frac{i}{2} \partial_{\mu} \otimes \theta^{\mu \nu}} \partial_{\nu}\left\{\delta_{u,-1} \otimes \mathbb{1}\right\} \quad .
$$

as follows in the standard manner from the coproduct. It is easy to check that this product is not coassociative. The calculation is similar to the one leading to (5.7) Thus

$$
\begin{gathered}
e_{p} *\left(e_{q} * e_{r}\right) \neq\left(e_{p} * e_{q}\right) * e_{r} \\
e_{p} \in \mathcal{B}_{\theta}^{+1}\left(\mathbb{R}^{N}\right), \quad e_{q, r} \in \mathcal{B}_{\theta}^{-1}\left(\mathbb{R}^{N}\right) .
\end{gathered}
$$

The loss of associativity also follows using general considerations and the nonassociativity of the coproduct [17-19].

\section{$7 \quad$ The Quasi-Hopf Structure of $\overline{\hat{\mathcal{P}}_{+}^{\uparrow}}$}

We have a new coproduct on $\overline{\hat{\mathcal{P}}_{+}^{\uparrow}}$ which is obtained by twisting with the twist element $\overline{\mathfrak{F}_{\theta}}$. For this twist to generate a Hopf algebra, it must satisfy (2.11), which it does, owing to the fact that

$$
\epsilon\left(P_{\mu}\right)=0
$$


Now we also saw that the resulting coproduct $\overline{\Delta_{\theta}}$ is not coassociative. It means that the resultant Hopf algebra is only a quasi-Hopf algebra.

Indeed, for the twist to generate a Hopf algebra, it must satisfy (2.12). But we can show that the twist element $\overline{\mathfrak{F}_{\theta}}$ does not satisfy it. By simple algebra one can calculate that

$$
\begin{gathered}
\left(\overline{\mathfrak{F}_{\theta}} \otimes \mathbb{1}\right)\left(\Delta_{0} \otimes i d\right) \overline{\mathfrak{F}_{\theta}}=\exp \left[-\frac{i}{2} \theta^{\mu \nu}\left(\left(P_{\mu} \otimes \mathbb{1} \otimes P_{\nu}+\mathbb{1} \otimes P_{\mu} \otimes P_{\nu}\right)\left(\delta_{u \otimes u,-1} \otimes \mathbb{1}\right)\right.\right. \\
\left.\left.+\left(P_{\mu} \otimes P_{\nu} \otimes \mathbb{1}\right)\left(\delta_{u,-1} \otimes \mathbb{1} \otimes \mathbb{1}\right)\right)\right] .
\end{gathered}
$$

On the other hand

$$
\begin{gathered}
\left(\mathbb{1} \otimes \overline{\mathfrak{F}_{\theta}}\right)\left(i d \otimes \Delta_{0}\right) \overline{\mathfrak{F}_{\theta}}=\exp \left[-\frac{i}{2} \theta^{\mu \nu}\left(\left(P_{\mu} \otimes P_{\nu} \otimes \mathbb{1}+P_{\mu} \otimes \mathbb{1} \otimes P_{\nu}\right)\left(\delta_{u,-1} \otimes \mathbb{1} \otimes \mathbb{1}\right)\right.\right. \\
\left.\left.+\left(\mathbb{1} \otimes P_{\mu} \otimes P_{\nu}\right)\left(\mathbb{1} \otimes \delta_{u,-1} \otimes \mathbb{1}\right)\right)\right] .
\end{gathered}
$$

It is clear that (7.2) and (7.3) are not equal. The terms involving $\delta_{(u \otimes u),-1}$ in (7.2) are absent in (17.3). Hence

$$
\mathbb{1} \otimes \overline{\mathfrak{F}_{\theta}}\left(i d \otimes \Delta_{0}\right) \overline{\mathfrak{F}_{\theta}} \neq \overline{\mathfrak{F}_{\theta}} \otimes \mathbb{1}\left(\Delta_{0} \otimes i d\right) \overline{\mathfrak{F}_{\theta}}
$$

Thus this twist does not give an ordinary Hopf algebra. But we do get a quasi-Hopf algebra. For as we explained in section 2, all one needs is the property (2.11) to get a quasi-Hopf algebra.

Actually if (2.12) were satisfied, $\overline{\Delta_{\theta}}$ would have been coassociative. Its failure proved in section 5 thus already shows that (2.12) is not fulfilled.

\section{Final Remarks}

We have shown the existence of a quasi-Hopf symmetry structure in a quantum gauge field theory where only matter fields feel the noncommutativity of spacetime. The coproduct is not coassociative and the $*$-product on the (two-sheeted) spacetime is not associative in

such a theory int the presence of both matter and gauge fields.

\section{Acknowledgements}

We want to thank Alberto Ibort and the Universidad Carlos III de Madrid for their wonderful hospitality and support.

A.P.B. thanks T. R. Govindarajan and the Institute of Mathematical Sciences, Chennai for very friendly hospitality as well.

A.P.B. is most grateful to Mario Martone for help in preparing the manuscript.

The work of A.P.B.was supported in part by DOE under the grant number DE-FG0285ER40231 and by the Department of Science and Technology, India.

The work of B.Q. was supported by IRCSET fellowship and by Perimeter Institute. 


\section{References}

[1] R. Oeckl, "Untwisting noncommutative $\mathbb{R}^{d}$ and the equivalence of quantum field theories", Nucl. Phys. B581:559-574 [arXiv:hep-th/003018]; M. Chaichian, P. P. Kulish, K. Nishijima and A. Tureanu, "On a Lorentz-invariant interpretation of noncommutative space-time and its implications on noncommutative QFT," Phys. Lett. B 604, 98 (2004) arXiv:hep-th/0408069.

[2] M. Dimitrijevic and J. Wess, "Deformed bialgebra of diffeomorphisms," arXiv:hep-th/0411224.

[3] P. Aschieri, C. Blohmann, M. Dimitrijevic, F. Meyer, P. Schupp and J. Wess, "A gravity theory on noncommutative spaces," Class. Quant. Grav. 22, 3511 (2005) arXiv:hep-th/0504183.

[4] H. Grosse, "On The Construction of Möller Operators for the Nonlinear Schrödinger Equation," Phys. Lett. B 86, 267 (1979).

[5] A. B. Zamolodchikov and A. B. Zamolodchikov, "Factorized S-matrices in two dimensions as the exact solutions of certain relativistic quantum field models," Annals Phys. 120, 253 (1979).

[6] L. D. Faddeev, "Quantum completely integral models of field theory," Sov. Sci. Rev. C 1, 107 (1980).

[7] A. P. Balachandran, A. Pinzul and B. A. Qureshi, "UV-IR Mixing in NonCommutative Plane," Phys. Lett. B 634, 434 (2006) arXiv:hep-th/0508151.

[8] A. P. Balachandran, T. R. Govindarajan, G. Mangano, A. Pinzul, B. A. Qureshi and S. Vaidya, "Statistics and UV-IR mixing with twisted Poincare invariance," Phys. Rev. D 75, 045009 (2007) arXiv:hep-th/0608179.

[9] D. Buchholz and S. J. Summers, "Warped Convolutions: A Novel Tool in the Construction of Quantum Field Theories," arXiv:0806.0349 [math-ph].

[10] D. Vassilevich, "Twist to close", Mod. Phys. Lett. A21:1279-1284 (2006), arXiv:hep-th/0602185]; P. Aschieri, M. Dimitrijevic, F. Meyer, S. Schraml and J. Wess, "Twisted gauge theories," Lett. Math. Phys. 78, 61 (2006) arXiv:hep-th/0603024.

[11] D. Vassilevich, "Symmetries in noncommutative field theories: Hopf versus Lie", [arXiv:hep-th/0711.4091].

[12] A. P. Balachandran, A. Pinzul, B. A. Qureshi and S. Vaidya, "Twisted Gauge and Gravity Theories on the Groenewold-Moyal Plane," Phys. Rev. D 76, 105025 (2007) [arXiv:hep-th/0708.0069].

[13] A. P. Balachandran, A. Pinzul, B. A. Qureshi and S. Vaidya, "S-Matrix on the Moyal Plane: Locality versus Lorentz Invariance," Phys. Rev. D 77, 025020 (2008) arXiv:0708.1379 [hep-th]], 0708.0069. 
[14] E. Akofor, A. P. Balachandran, S. G. Jo and A. Joseph, "Quantum fields on the Groenwold-Moyal plane: C, P, T and CPT", Phys. Rev. D77:105032 (2008) arXiv:0706.1259].

[15] A. P. Balachandran, A. Pinzul and A. R. Queiroz, "Twisted Poincaré Invariance, Noncommutative Gauge Theories and UV-IR Mixing", Phys. Lett. B668:241-245 (2008) arXiv:0804.3588.

[16] Anosh Joseph, "Particle phenomenology on noncommutative spacetime", arXiv:0811.3972.

[17] G. Mack and V. Schomerus, "Quantum symmetry in quantum theory," Cargese 1991: 0329-353.

[18] G. Mack and V. Schomerus, "QuasiHopf quantum symmetry in quantum theory," Nucl. Phys. B 370, 185 (1992).

[19] G. Mack and V. Schomerus, "Quantum symmetry for pedestrians," DESY-92-053, March 1992.

[20] S. Majid, "Foundations of Quantum Group Theory", Cambridge University Press, 1995.

[21] A. P. Balachandran, T. R. Govindarajan and S. Vaidya, "Spontaneous Symmetry Breaking in Twisted Noncommutative Quantum Theories," [arXiv:hep-th/0901.1712].

[22] A. P. Balachandran and M. Martone, Mod. Phys. Lett. A 24, 1811 (2009) arXiv:0902.3409 [hep-th]].

[23] A. P. Balachandran, A. Pinzul and B. A. Qureshi, "Twisted Poincaré Invariant Quantum Field Theories," Phys. Rev. D 77, 025021 (2008) [arXiv:0708.1779 [hep-th]]. 